\title{
Cloud-Based NAS (Network Attached Storage) Analysis as an Infrastructure as A Service (IAAS) Using Open Source NAS4FREE and Owncloud
}

\author{
Hariyo Sasongko ${ }^{1}$, T.Yudi Hadiwandra ${ }^{2}$ \\ Informatics Engineering, Engineering Faculty, Riau University \\ hariyo.sasongko2326@student.unri.ac.id ${ }^{1}$,tydihw@ lecturer.unri.ac.id ${ }^{2}$
}

\section{Article Info}

History :

Received Oct 21, 2020

Revised May 06, 2021

Accepted Sept 16, 2021

Keywords :

Cloud Computing

NAS

NAS4FREE

\begin{abstract}
At this time, computer systems and networks are an essential part of human life. The number can see this of computer users in the office or on campus, and at school. This need has reached a relatively large number. To access data, many computer users in the network continue to increase, impacting the selection of servers, and large data storage media is necessary. Currently, the storage media that is often used to store data still uses physical media such as hard disks, flash disks that are virus-prone and easy to lose. Another problem also arises when physical storage has a relatively high price compared to cloud storage that can be accessed anywhere when needed.
\end{abstract}

(C) This work is licensed under a Creative Commons AttributionShareAlike 4.0 International License.

Corresponding Author:

Hariyo Sasongko

Informatics Engineering, Engineering Faculty

Riau University

Bina Widya Km.12,5 Simpang Baru, Pekanbaru, Indonesia, 28293

Email : Hariyo.sasongko2326@ student.unri.ac.id

\section{INTRODUCTION}

At this time, computer systems and networks are an essential part of human life. This can be seen by the number of computer users in offices, companies, factories, or even on campus and in schools. This need has reached a relatively large number. Humans usually use the computer as a daily operational tool or even a system that can help solve problems [1]. In accessing data, many individuals and organizational computer users in the network, which continues to increase, impact choosing a good server and large data storage media is essential. For now, the storage media that we often use to store data still use physical media such as hard disks and flash disks that are prone to viruses and are easily lost. Another problem also arises when physical storage media has a relatively high price. In contrast to the progress of storage media, which uses cloud storage technology that can be accessed wherever and whenever needed.

NAS (Network Attached Storage) is one solution to expensive storage media because the NAS does not require high hardware resources to share files [2]. NAS is a server with an operating system devoted to serving the needs of data files. This server is also in charge of providing services to other terminals connected to the network system or clients. The NAS can be accessed over the local network with a TCP/IP protocol.

A previous study conducted by Kartika Imam Santoso and Muhammad Abdul Muin entitled Implementation of Network Attached Storage (NAS) Using NAS4FREE for Media Backup Files. 
The study results provide an overview of how NAS4FREE is used as an operating system to be a backup media file and test the ability of NAS4FREE to upload and download. Then, Tajudin Akbar et al. conducted a Comparative Analysis of the Performance of FreeNAS and NAS4FREE as a Network Attached Storage (NAS) Network Operating System on a Local Area Network (LAN). In the study, the two NAS systems showed relatively the same level of performance for testing file copy and duplicate file detection; the overall test results can be concluded that the processor's clock speed on each client has a significant effect on the test. The faster the clock speed of a client, the quicker the rate of detecting file detection and copying files. In this study, researchers will use the NAS4FREE operating system to build a NAS server, and researchers will also use their cloud as a shared storage medium that users can access [3].

\section{RESEARCH METHOD}

The methodology used in this study is described in a flowchart, as shown in Figure 1 below.

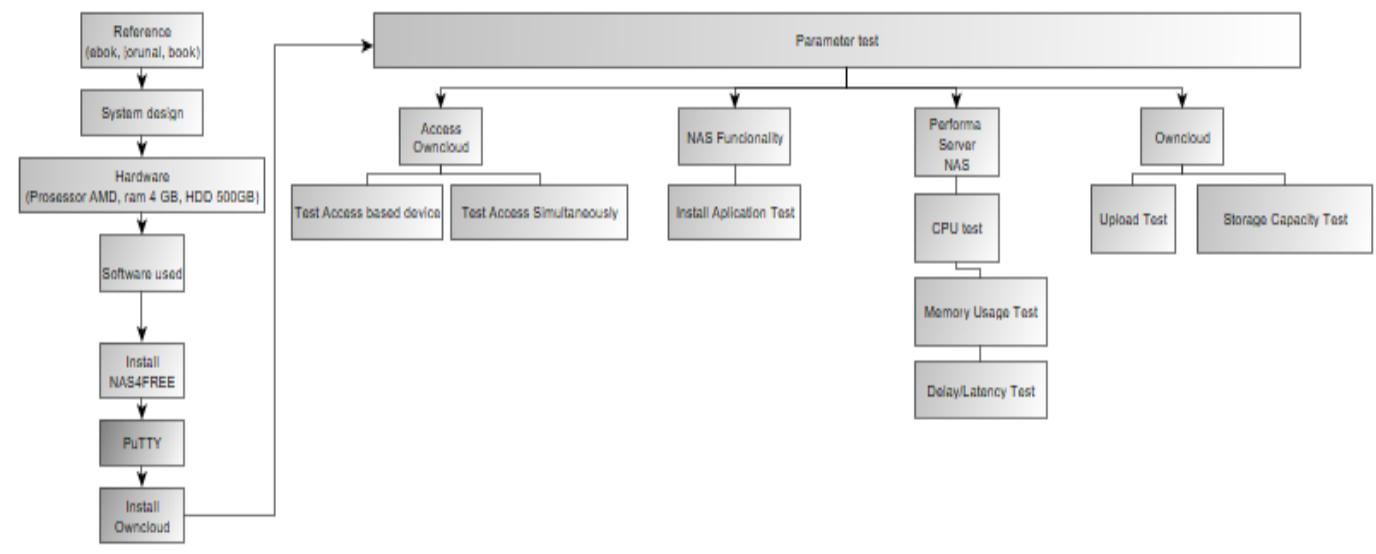

Figure 1. Research Methodology

In Figure 1 above, it can be explained how the research method used in this study, where the research begins by collecting data through journals, books, or ebooks and continues with designing a cloud storage system using its cloud. After that, the researchers also observed what software would be used in this study. The next stage is to install the server that will be used, which. In this study, the researcher uses the NAS4FREE operating system and continues to install owncloud via putty; if everything is installed, then do the test using the parameters determined previously.

\subsection{Data Collection}

The data collection method used in this study is by observing the high cost of storage media, both current storage media, both physical and online, or commonly known as cloud computing. Then the researchers also took a library research approach, namely collecting data from various expert opinions from scientific journals and books related to research material.

\subsection{Theory}

Cloud Computing is a model for enabling easy, on-demand network access to a shared set of configurable compute resources, e.g. (network, servers, storage, applications, and services) that can be rapidly provisioned and released with minimal management effort or NIST provider interaction. Cloud Storage is an inseparable part of cloud computing, used in data storage by utilizing thirdparty servers as service providers. At the time of data storage, the user sees a virtual server, where the place or location of the server is not known private cloud or internal cloud is a cloud computing service offered in a private network. Its products include virtualization automation. This product provides the ability to host applications or virtual machines on enterprise hosts [4]. 
OwnCloudis one of the platforms of cloud computing technology that is free and easy to develop (open source).]. NAS (Network Attached Storage)is a data storage medium that can be accessed via a computer network (TCP/IP) and not directly connected to the host. Several hosts can share one storage media; with this, the cost of processing storage media on each host can be reduced. NAS uses the NFS (Network File System) protocol. A NAS can be viewed as a network drive and can be used to store documents and files. A NAS is a computer op-timed in hardware and software to become a file server [6]. Delay (latency) measures the time it takes for data to travel from origin to destination. Delay can be affected by space, physical media, congestion, or a lengthy process [7]. The formula for calculating the delay value is:

$$
\text { Average delay }=\text { Total delay } / \text { Total packets received }
$$

Based on TIPHON's standard, the amount of delay can be classified as follows:

Table 1. Standard Delay/latency

\begin{tabular}{cc}
\hline Latency Category & Big Delay \\
\hline Very good & $<150 \mathrm{~ms}$ \\
Good & 150 to $300 \mathrm{~ms}$ \\
Currently & 300 to $450 \mathrm{~ms}$ \\
Bad & $>450 \mathrm{~ms}$ \\
\hline
\end{tabular}

\subsection{Software dan Hardware}

The hardware used in this study is as shown in table 2 below:

Table 2. Hardware

\begin{tabular}{|c|c|}
\hline Servers & Specification \\
\hline 1 PC Asus Laptop & $\begin{array}{c}\text { Processor AMD } \\
\text { Dual-Core A9- } \\
9420 \\
\text { Memory 4GB } \\
\text { HDD 500GB } \\
\end{array}$ \\
\hline Client & Specification \\
\hline $\begin{array}{l}1 \text { Piece Smartphone Samsung } \\
\text { A20S }\end{array}$ & $\begin{array}{c}\text { Android Pie } \\
\text { Operating System } \\
(9.0) \\
\text { 3GB RAM }\end{array}$ \\
\hline
\end{tabular}

The software used in this study is as shown in table 3 below:

Table 3. Material

\begin{tabular}{cc}
\hline Software Name & Function \\
\hline NAS4FREE-x64-LiveCD-11.1 & As a NAS \\
& $\begin{array}{c}\text { Network Attached } \\
\text { Storage) Server } \\
\text { Operating System }\end{array}$ \\
\hline OwnCloud10.3.1 & As a SAN (Storage \\
& Area Network) \\
& Platform \\
\hline Virtualbox & As a Virtual \\
PuTTY & Machine \\
\hline \multirow{2}{*}{ Ps remote access } \\
server
\end{tabular}




\begin{tabular}{cc}
\hline Observium16.0 - AMD64 & $\begin{array}{c}\text { As a Memory } \\
\text { usage and CPU } \\
\text { usage tester }\end{array}$ \\
\hline Wireshark- win64-3.2.4 & $\begin{array}{c}\text { As a delay } \\
\text { (latency) tester }\end{array}$ \\
\hline Monitor Bandwidth & $\begin{array}{c}\text { As a tester, upload } \\
\text { and download files }\end{array}$ \\
\hline oCloud.de & $\begin{array}{c}\text { To access } \\
\text { OwnCloud service } \\
\text { via smartphone }\end{array}$ \\
Owncloud Client & To access \\
& OwnCloud \\
& services via \\
desktop
\end{tabular}

\subsection{Test Scenario}

The test scenario in this study consisted of 4 tests, namely testing cloud storage performance by testing access to own cloud services through desktop and smartphone-based devices. The cloud storage performance test flowchart can be seen in Figure 2.

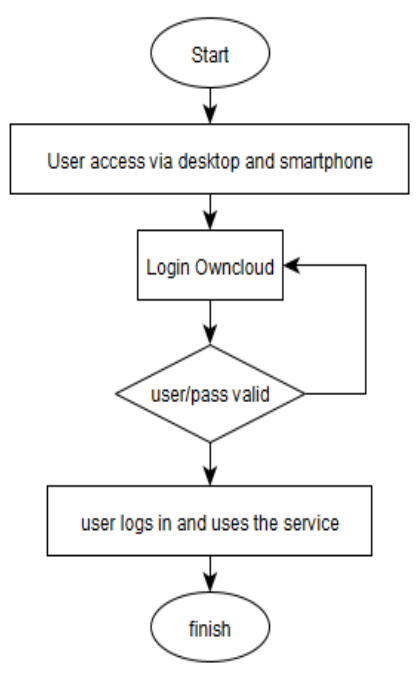

Figure 2. Flowchart of own cloud service access test

NAS functionality testing is carried out to determine whether the NAS can provide storage services on the network as if it were on a local PC. NAS functionality testing flowchart can be seen in Figure 3. 


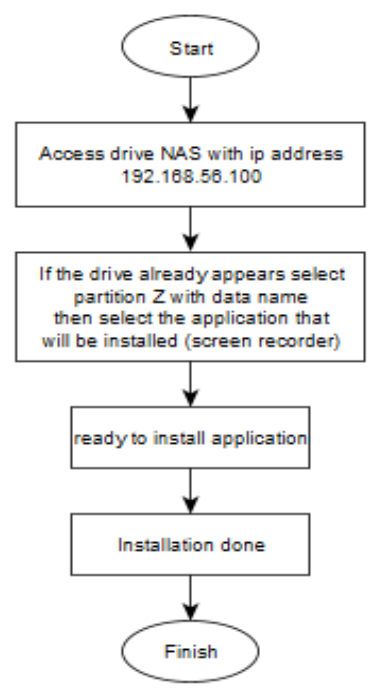

Figure 3. NAS functionality testing flowchart

Furthermore, testing the performance of the NAS virtual server in this case, NAS4FREE is used as a virtual server operating system; the things tested include CPU usage testing, memory usage testing, and delay/latency testing. The server performance testing flowchart can be seen in Figure 4.

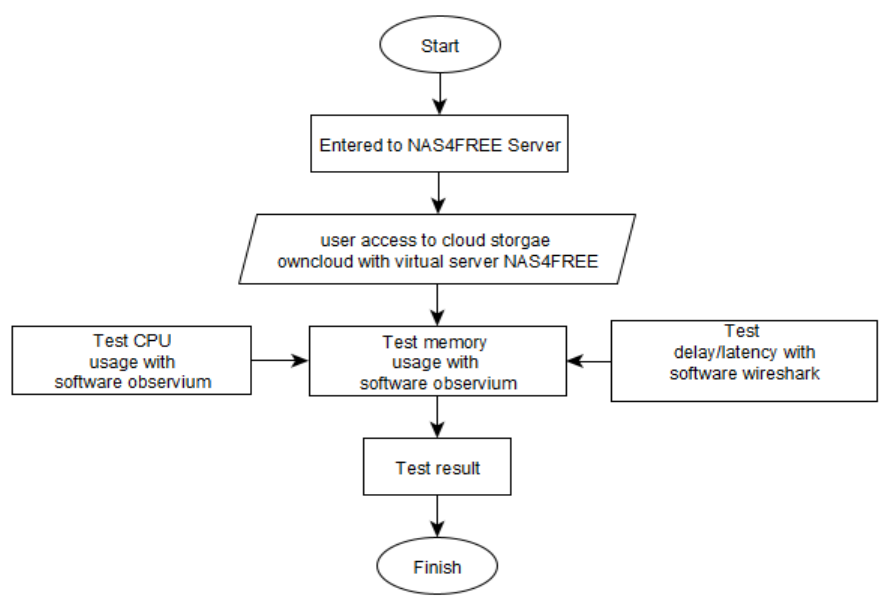

Figure 4. NAS4FREE virtual server test flowchart

Some of the test parameters are Testing uploads and downloading files with various file sizes to the Owncloud service. Then there will also be a test of the existing storage capacity on the Owncloud user, using the maximum power of the own cloud, which will be tested at 5 GB. The own cloud performance testing flowchart can be seen in Figures 5 and 6. Figure 5 is the process to try the upload and download of several files to a cloud service. 


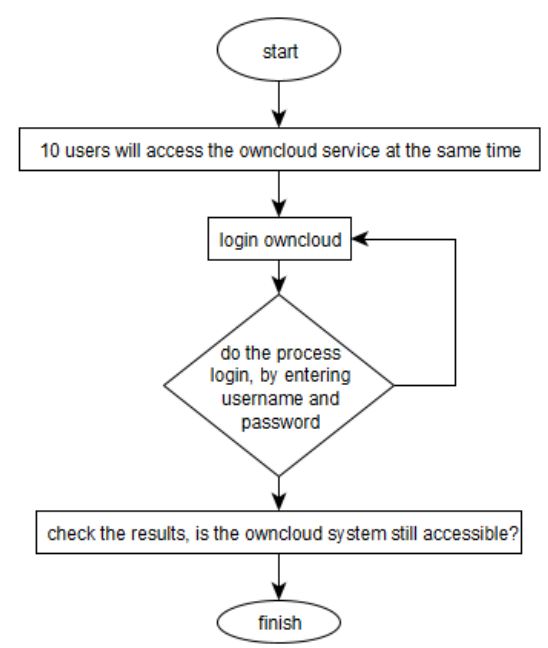

Figure 5. Flowchart of upload and download test files

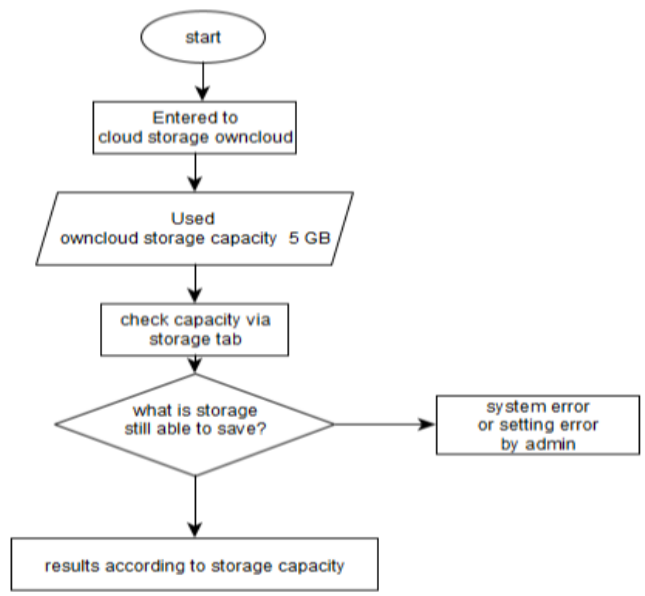

Figure 6. Storage Capacity Test

It can be seen in Figure 6 above that the storage capacity test process will be carried out by uploading files to cloud services up to the maximum storage limit.

\section{RESULT AND DISCUSSION}

\subsection{System Test}

At the system testing stage, researchers will conduct tests with several parameters, namely;

1. Performance testing of cloud storage by testing client access via smartphone and PC-based devices.

2. NAS functionality testing.

3. Testing the performance of the NAS virtual server, in this case using NAS4FREE as the operating system; the things to be tested include testing CPU usage performance, trying memory usage, and testing delay/latency.

4. Performance testing of the OwnCloud platform by testing several parameters, namely, file upload and download tests and storage capacity tests.

\subsubsection{Cloud Storage Testing Performance}

Performance testing of cloud storage is a method of testing access from 2 different devices, namely smartphones and PC-based devices. This test focuses on the access side, whether the system from the cloud storage that has been built can be accessed.

Hariyo, Analysis of Cloud Based NAS (Network Attached Storage) As Infrastucture as a Service (IAAS) 


\section{Access Desktop Testing}

Run the OwnCloud client application, fill in the server IP address: port/own cloud; in this study, the server IP address uses IP 192.168.56.100 with port 8080 and /own cloud. If it is, fill in the account username and password, then click next; if successfully logged in, OwnCloud will synchronize with the user's PC; if successful, the own cloud desktop display will be as shown in Figure 7.

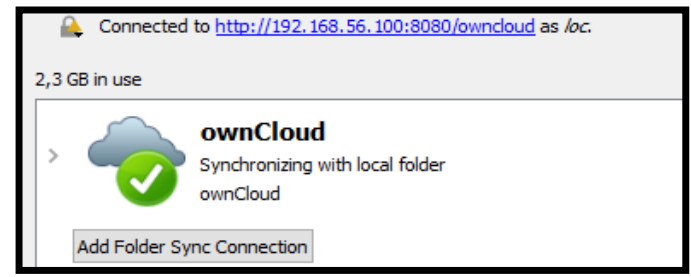

Figure 7. Owncloud sync process

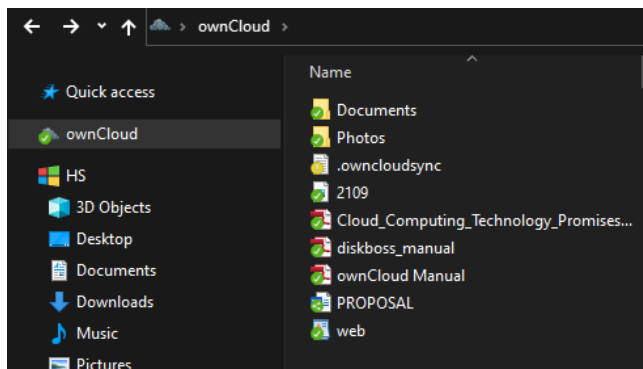

Figure 8. Successfully synced files to own cloud desktop

\section{Smartphone Access Testing}

Before testing access via a smartphone, make sure you have downloaded and installed the oCloud.de application on the Google Play Store. Once installed, enter the IP address of the OwnCloud server; in this study, the server's IP address is 192.168 .56 .100 with port 8080 and /own cloud. If so, then fill in the account username and password and press connect to the OwnCloud service. The process of accessing owncloud via a smartphone can be seen in Figure 16.

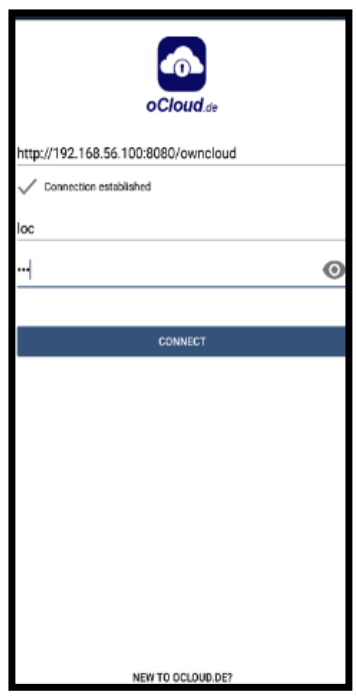

Figure 9. Owncloud login process via a smartphone 


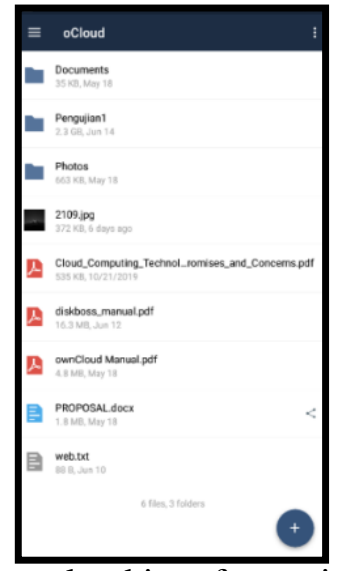

Figure 10. Owncloud interfaces via smartphone

3. Simultaneously Access Testing

Testing access to the own cloud storage service simultaneously by 10 users aims to see whether the number of users accessing the service affects system performance. Here are the results of concurrent access testing. Before accessing, here is a list of 10 users who will access Owncloud.

After testing access by 10 users simultaneously, the test results are obtained as follows: When the access test is carried out, where when users 8,9 and 10 are about to access, the server suddenly restarts itself, this makes users 8.9 and 10 cannot access Owncloud services for a while, but this can be overcome by continuing the cloud storage server.

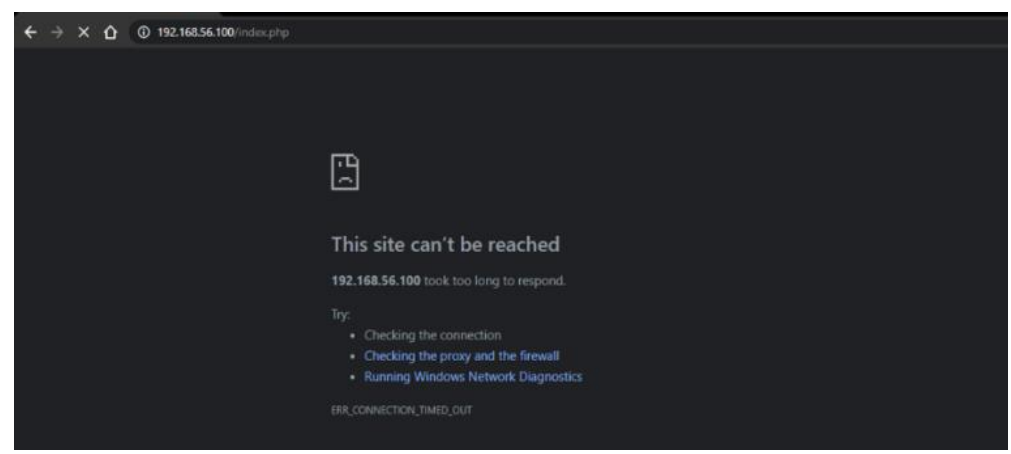

Figure 11. The server restarts by itself

Table 4. Concurrent access test results

\begin{tabular}{cc}
\hline Username & Test results \\
\hline User 1 & Succeed \\
\hline User 2 & Succeed \\
\hline User 3 & Succeed \\
\hline User 4 & Succeed \\
\hline User 5 & Succeed \\
\hline User 6 & Succeed \\
\hline User 7 & Succeed \\
\hline User 8 & $\begin{array}{c}\text { It worked after } \\
\text { the server was } \\
\text { restarted }\end{array}$ \\
\hline
\end{tabular}

Hariyo, Analysis of Cloud Based NAS (Network Attached Storage) As Infrastucture as a Service (IAAS) Using Open Source Nas4free and Owncloud 


\begin{tabular}{cc}
\hline User 9 & $\begin{array}{c}\text { It worked after } \\
\text { the server was } \\
\text { restarted }\end{array}$ \\
\hline Users 10 & $\begin{array}{c}\text { It worked after } \\
\text { the server was } \\
\text { restarted }\end{array}$ \\
\hline
\end{tabular}

\subsubsection{NAS Functionality Test}

NAS functionality testing is carried out to determine whether the NAS can provide storage services on the network as if it were on a local PC. The test is done by connecting the storage on the NAS with a local PC so that the hold on the NAS looks like it's on a local PC. then to prove that the files in the NAS storage can be directly used as the files in the local storage, the researcher will install the applications that are on the NAS storage to a local PC. The results of testing the NAS functionality can be seen in Figures 12 and 13.

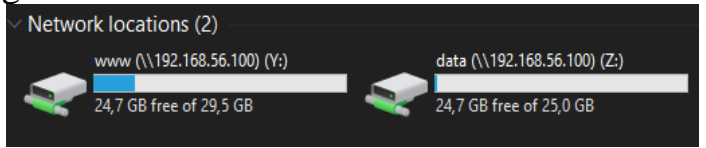

Figure 12. NAS drive on local PC

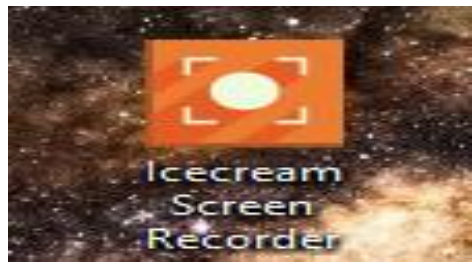

Figure 13. Successfully installed applications

\subsubsection{NAS Performance Testing}

NAS performance testing is a test method where this test is carried out to test the strength of a virtual server that has been built using the NAS4FREE operating system.

1. CPU Usage Testing

CPU usage testing is done by copying files from the client to the server. Testing is done by placing 50 different files, and the extensions are exe, jpg, $\mathrm{mp} 3$, and $\mathrm{mp} 4$. This test focuses on testing how much CPU usage is done when copying files. Testing is done using observe software to monitor CPU usage. The results of the CPU usage test can be seen in Figure 14.

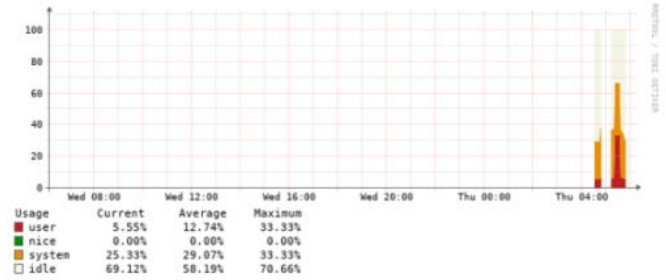

Figure 14. CPU usage results on the NAS4FREE server

The results of the CPU usage test are shown in Figure 14 by copying files from the client to the server; the graph on the observe shindicateshat the CPU usage on the NAS4FREE server is $33.33 \%$, with an average user of $12.74 \%$.

\section{Memory Usage Testing}

SiLikePU usage testing, memory usage testing is also done by copying files from the client to the server. The test is done by placing 50 files with different file extensions, and the extensions are exe, jpg, mp3, and mp4. This test focuses on testing how much memory is used when copying files. 
Testing is done using observe software to monitor memory usage. Memory usage test results can be seen in Figure 15.

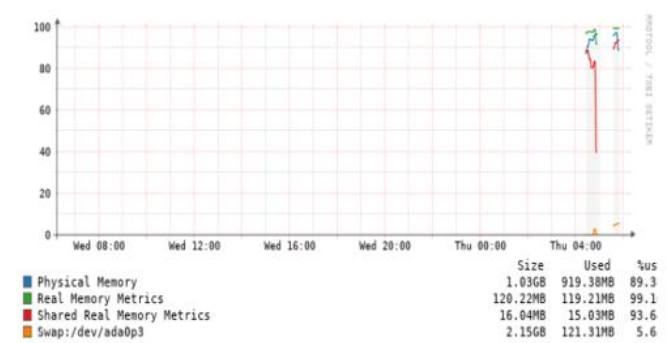

Figure 15. Memory usage test results on the NAS4FREE server

The results of the memory usage test are shown in Figure 15 by copying files from the client to the server; the graph on the observium indicates that the memory usage on the NAS4FREE server is $919.38 \mathrm{MB}$ from a memory size of $1.03 \mathrm{~GB}$ with a usage percentage of $89.3 \%$.

3. Delay/latency testing

Delay/latency testing is done by sending a packet between clients; the focus of this test is to see how long it takes a package or data to reach the client. Testing is done using Wireshark software.

\begin{tabular}{|llll|}
\hline Statistics & & & \\
Measurement & $\frac{}{\text { Captured }}$ & Displayed & Marked \\
\hline Packets & 443 & $443(100.0 \%)$ & - \\
Time span, s & 106.507 & 106.507 & - \\
Average pps & 4.2 & 4.2 & - \\
Average packet size, B & 356 & 356 & - \\
Bytes & 157923 & $157923(100.0 \%)$ & - \\
Average bytes/s & 1482 & 1482 & - \\
Average bits/s & $11 \mathrm{k}$ & $11 \mathrm{k}$ & - \\
\hline
\end{tabular}

Figure 16. The results of the delay/latency test using Wireshark

From the data capture that has been done with Wireshark, the results are as shown in Figure 16, then the way to calculate the average delay is to use the formula:

$$
\begin{aligned}
\text { Average delay }= & \frac{\text { Total Delay }}{\text { Total package recieve }} \\
& =\frac{106.507}{443} \\
=0.2404221219 \text { second } & \\
& =0.2404221219 \times 1000 \\
& =240.4221219 \text { millisecond }(\mathrm{ms})
\end{aligned}
$$

\subsubsection{Owncloud Platform Testing}

OwnCloud platform testing is a test method where this test is carried out to test the functionality of a software application.

1. Upload file testing

File upload test results are displayed in tabular form. Table 5 shows the results of testing the file upload test.

Table 5. Results of the file upload test

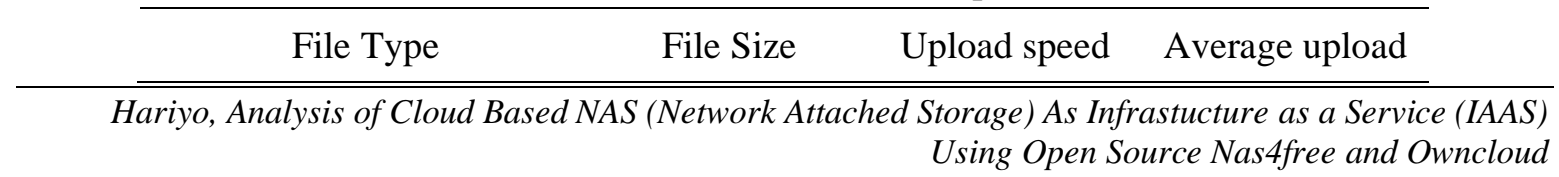




\begin{tabular}{cccc}
\hline MP4 & $4.87 \mathrm{MB}$ & $2.28 \mathrm{MB} / \mathrm{s}$ & $118.0 \mathrm{~KB} / \mathrm{s}$ \\
MP3 & $7.37 \mathrm{MB}$ & $2.28 \mathrm{MB} / \mathrm{s}$ & $72.2 \mathrm{~KB} / \mathrm{s}$ \\
JPG & $1.23 \mathrm{MB}$ & $5.44 \mathrm{MB} / \mathrm{s}$ & $124.8 \mathrm{~KB} / \mathrm{s}$ \\
EXE & $1.16 \mathrm{MB}$ & $1.27 \mathrm{MB} /$ & $27.7 \mathrm{~KB} / \mathrm{s}$ \\
\hline
\end{tabular}

2. Download file testing

The test results of downloading files are displayed in tabular form. Table 6 shows the results of testing the file download test.

Table 6. Results of the file download test

\begin{tabular}{cccc}
\hline File Type & File Size & $\begin{array}{c}\text { Download } \\
\text { speed }\end{array}$ & $\begin{array}{c}\text { Average } \\
\text { download }\end{array}$ \\
\hline MP4 & $3.32 \mathrm{MB}$ & $7.27 \mathrm{MB} / \mathrm{s}$ & $701.1 \mathrm{~KB} / \mathrm{s}$ \\
MP3 & $3.30 \mathrm{MB}$ & $2.96 \mathrm{MB} / \mathrm{s}$ & $73.3 \mathrm{~KB} / \mathrm{s}$ \\
JPG & $3.00 \mathrm{MB}$ & $2.94 \mathrm{MB} / \mathrm{s}$ & $54.7 \mathrm{~KB} / \mathrm{s}$ \\
EXE & $2.01 \mathrm{MB}$ & $2.09 \mathrm{MB} / \mathrm{s}$ & $61.2 \mathrm{~KB} / \mathrm{s}$ \\
\hline
\end{tabular}

\section{Storage capacity testing}

Storage capacity testing is a test of the maximum capacity of the storage media contained in Owncloud. The test is carried out using the total capacity of Owncloud storage, tested at 5 GB. Then the results will be seen, whether by the storage capacity that has been provided, which in this test is 5 GB.

After testing, it was found that the storage on its cloud was by the capacity given by the admin as a storage media manager. This can be seen in Figure 17 below.

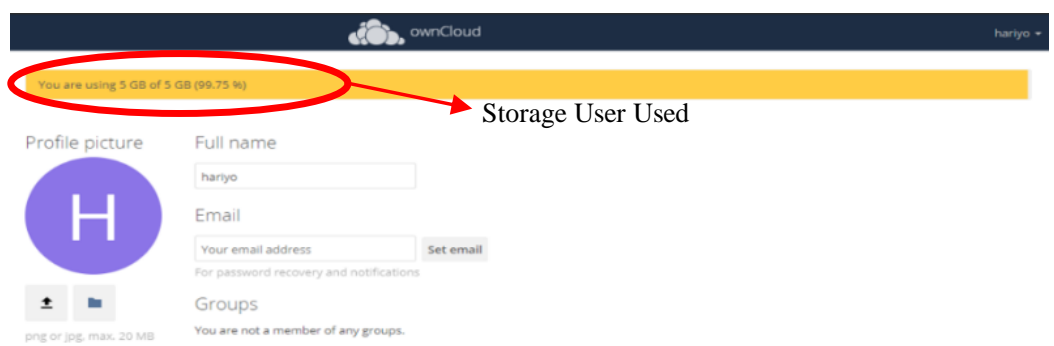

Figure 17. Storage capacity test results

\subsection{Data Analyze}

In general, data analysis is a process or effort in processing data into new information so that the characteristics of the data become easier to understand and valuable as a solution to a problem, primarily related to research.

From the research that has been done, the data obtained from various tests that have been carried out, and the data will be analyzed as follows:

1. Access test analysis by device and access testing concurrently

The OwnCloud access test through the desktop shows that the desktop device successfully accesses OwnCloud and performs the data synchronization process on the user's PC. Data synchronization aims to synchronize files on the OwnCloud website, which will then be synchronized with the user's PC, making it easier to access the files.

From the results of testing access to the Owncloud cloud storage service simultaneously, which was carried out by 10 users who aim to see if there is an effect on the system when accessing it simultaneously, the results show that user 1 to user 7 can access the own cloud service. Still, when user 8 to user 10 is about to access, the server suddenly restarts by itself. Still, users 8 to 10 could access their cloud service after the server was running again after restarting. This shows that the cloud server has not handled access requests by 10 users at once. 
2. NAS functional testing

From the results of the NAS functional testing that has been carried out, the results show that the NAS drive located on the network can be accessed like a local drive on a PC and the installation of applications stored on the NAS drive shows that files stored on the NAS drive can be directly run or installed like files held on a local PC without having to download it first.

\section{CPU Usage Analyze}

The results of testing CPU usage using observium software found that CPU usage on the "virtual server," which in this study used the NAS4FREE operating system built on the Virtualbox platform, was $33.33 \%$, with an average usage of $12.74 \%$. Then the average CPU usage results on one day of use, which is $20.0 \%$, and the average usage is $8.4 \%$.

In a previous study, conducted by Tajudin Akbar et al. (2014), who also examined the performance of NAS4FREE as a NAS server operating system using a physical device that was used as a NAS server by testing file copies from the client to the server in the results of his research NAS4FREE CPU usage used resources of $29.50 \%$. When compared with the results of research conducted by Tajudin Akbar et al. (2014), which uses a physical server as the media server and the tests carried out by the author, it can be concluded that CPU usage performance is better using a physical media server compared to using a virtual server in the study. This time the author uses Virtualbox to build a NAS server.

\section{Memory Usage Analyze}

From the results of the Memory usage test using observium software, it was found that the memory usage on the "virtual server," which in this study used the NAS4FREE operating system built on the Virtualbox platform, was $919.38 \mathrm{MB}$ from a memory size of $1.03 \mathrm{~GB}$ with a usage percentage of $89.3 \%$. Then the maximum result of the average CPU usage on one day of use is $965.03 \mathrm{MB}$, and the average usage is $949.35 \mathrm{MB}$ with a usage percentage of $96 \%$.

In a previous study, conducted by Tajudin Akbar et al. (2014), who also examined the performance of NAS4FREE as a NAS server operating system using a physical device that was used as a NAS server by testing copy files from the client to the server in the results of his research NAS4FREE memory usage uses resources of $165.99 \mathrm{MB}$. When compared with the results of research conducted by Tajudin Akbar et al. (2014), which uses a physical server as the media server and the tests conducted by the author, it can be concluded that memory usage performance is better using a physical media server compared to using a virtual server in the study. This time the author uses Virtualbox to build a NAS server.

\section{Delay/latency Analyze}

The formula calculates the average delay using the results obtained are delays of 240.4221219 milliseconds (ms). With these results and based on the standard hold from TIPHON, the number is classified into the "good" category because the delay value is still in the range of 150 to 300 ms.

\section{Upload file Analyze}

From the test results of uploading files with the uploaded file types, namely MP4, MP3, JPG, and EXE, the MP4 file type with a file size of $4.87 \mathrm{MB}$, the average upload speed is $118.0 \mathrm{~KB} / \mathrm{s}$, and the maximum upload speed is $2.28 \mathrm{MB} / \mathrm{s}$. There is an MP3 file with a file size of $7.37 \mathrm{MB}$ with an average upload speed of $72.2 \mathrm{~KB} / \mathrm{s}$ and a full upload speed of $2.28 \mathrm{MB} / \mathrm{s}$. Furthermore, the JPG file size is $1.23 \mathrm{MB}$, the average upload is $124.8 \mathrm{~KB} / \mathrm{s}$, and the maximum upload speed is $5.44 \mathrm{MB} / \mathrm{s}$, and for EXE files with a file size of $1.16 \mathrm{MB}$, the average upload speed is $27.7 \mathrm{~KB} / \mathrm{s}$. With a full upload speed of $1.27 \mathrm{MB} / \mathrm{s}$. 
These test results found that the fastest file upload speed is in JPG-type files, with an average upload speed of $124.8 \mathrm{~KB} / \mathrm{s}$ and a maximum upload speed of $5.44 \mathrm{MB} / \mathrm{s}$ with a file size of 1.23. MB.

\section{Download file Analyze}

From the results of the test download files with the downloaded file types, namely MP4, MP3, JPG, and EXE, for the MP4 file type with a file size of $3.32 \mathrm{MB}$ with an average download speed of $701.1 \mathrm{~KB} / \mathrm{s}$ with a maximum download speed of 7.27 MB / s, then there are MP3 files with a file size of $3.30 \mathrm{MB}$ with an average download speed of $73.3 \mathrm{~KB} / \mathrm{s}$ and a maximum download speed of $2.96 \mathrm{MB} / \mathrm{s}$. Furthermore, for JPG files with a file size of $3.00 \mathrm{MB}$, the average download is $54.7 \mathrm{~KB} / \mathrm{s}$ with a maximum download speed of $2.94 \mathrm{MB} / \mathrm{s}$, and for EXE files with a length of $2.01 \mathrm{MB}$ average, Its download speed is $61.2 \mathrm{~KB} / \mathrm{s}$ with a full download speed of $2.09 \mathrm{MB} / \mathrm{s}$.

The test results found that the fastest file download speed is an MP4 file, with an average download speed of $701.1 \mathrm{~KB} / \mathrm{s}$ and a maximum download speed of $7.27 \mathrm{MB} / \mathrm{s}$ with a file size of 3.32 MB.

\section{Capacity Owncloud Storage Analysis}

From the results of testing the maximum capacity of storage media on own cloud, it was found that the limit size of the Hariyo user, which in this test the storage limit is set at 5GB, is by the limit on own cloud, the capacity setting or limitation on the user aims to provide storage capacity for each user. When using the owncloud cloud storage service, each user can adjust the storage capacity on their cloud according to needs. Here, the admin is responsible for managing the ability of each user.

\section{CONCLUSION}

Based on the test results in the research that has been done, it can be concluded that the access test based on the device was successfully carried out by accessing via smartphone and desktop devices, then simultaneously testing access, the user can access the own cloud service, but the virtual server restarts by itself, this shows that the server virtual has not been able to handle so much traffic. Testing the NAS functionality was successfully carried out by installing an application stored on the NAS drive. CPU usage on the NAS4FREE virtual server is $33.33 \%$, and memory usage is $919.38 \mathrm{MB}$ from a memory size of $1.03 \mathrm{~GB}$ with a usage percentage of $89.3 \%$. These results show that memory usage on NAS4FREE is relatively high.

He uploading test to the Owncloud service by uploading files with different file types showed that the JPG file was the fastest, with an average upload of $124.8 \mathrm{~KB} / \mathrm{s}$ and a maximum upload speed of $5.44 \mathrm{MB} / \mathrm{s}$ with a file size of $1.23 \mathrm{MB}$, to download files, the results are MP4 files, with an average download speed of $701.1 \mathrm{~KB} / \mathrm{s}$ and a maximum download speed of $7.27 \mathrm{MB} / \mathrm{s}$ with a file size of $3.32 \mathrm{MB}$. The storage capacity test was successfully carried out because it was by the capacity given by the admin, which was 5 GB.

For testing cloud storage built with conventional cloud storage, it can be concluded that traditional cloud storage has advantages in this regard, namely uploading and downloading files, with the average upload and download being higher than the own cloud storage.

The quality of a virtual cloud server built using Virtualbox is still far from being seen from the tests' results. With this, it is necessary to follow up to provide good service to users who will use this cloud storage service.

\section{REFERENCES}

[1] J. Miftahul, L. Baby, Basyah and AR Rizki, "Design of Network Attached Storage (NAS) on Raspberry Pi for Centralized Data Storage Based on WLAN," FIFO Scientific Journal, VoL. 7, No.2, November 2016.

[2] KI Santoso, and MA Muin, "Implementation of Network Attached Storage (NAS) Using 
NAS4Free for Media Backup Files," Scientific Journal of Informatics, 2(2), 123, 2016.

[3] Tajuddin, A., Jusak \& Teguh, S. "Comparative Analysis of the Performance of FreeNAS and NAS4FREE as Network Attached Storage (NAS) Network Operating Systems on Local Area Networks (LAN)". JISIKA, VoL.3, No.1(ISSN: 2338 -137X), 2015.

[4] NIST (National Institute of Standards and Technology), "Cloud Computing," A NIST Perspective and Beyond, Arlington VA, 2016.

[5] SS Choudari, and PR Chandre, "Study of Owncloud Replication of Mobile Information on Owncloud," Journal of Android and IOS Application and Testing, Vol.1, No.1, pp 2-3, 2016.

[6] NAS4FREE Team, "NAS4FREE Setup and User Guide,” 2019.

[7] Tiphon, "Telecommunication and Internet Protocol Harmonization Over Network (TIPHON) General Aspect of Quality of Service (QoS)," DTR/TIPHON-05006 (cb0010cs.PDF), 2017.

[8] Afrianto, I., Ahmad P. H. S., \& Sufa'atin, "Pemanfaatan Network Attached Storage (NAS) Sebagai Solusi Jaringan Small Office Home Office (SOHO)", Jurnal SENASKI (ISBN:978602-60250-1-2), 2017.

[9] Choudari, S.S, Chandre, P.R, "Study of Owncloud Replication of Mobile Information on Owncloud", Journal of Android and IOS Application and Testing, Vol.1, No.1. pp 2-3, 2017.

[10] Indrawata Wardhana, S. A, "Perancangan dan Penerapan Arsitektur Cloud Storage Pada Iain STS Jambi”, Manajemen Sistem Informasi, 2(1), 244-259. https://doi.org/http://dx.doi.org/10.11591/jurnalmsi.v12i4, 2017.

[11] Martanto, M, "Peningkatan Layanan Instansi Pendidikan Menggunakan Backup Data Dalam Upaya Mempermudah Akses Informasi. Jurnal Ilmiah Informatika", 7(01), 31. https://doi.org/10.33884/jif.v7i01.916, 2019.

[12] Sudetlin, S., Natasha, N. D., \& Darussalam, U, "Pemanfaatan Private Cloud Storage Berbasis Infrastructure As A Service (IAAS)", JOINTECS (Journal of Information Technology and Computer Science), 3(1). https://doi.org/10.31328/jointecs.v3i1.497, 2019.

[13] Tiphon, "Telecommunication and Internet Protocol Harmonization Over Network (TIPHON) General Aspects of Quality of Service (QoS)," DTR/TIPHON-05006 (cb0010cs.PDF), 2018.

[14] NIST (National Institute of Standards and Technology), "definition of Cloud Computing," NIST Special Publication 800-145, 2016.

[15] Koivisto, Jari-Pekka, "Network-Attached Storage For Small Companies. Degree Programme in Business Information Technology", Bachelor Thesis, Lathi University of Applied Sciences, Finland, 2016. 


\section{BIOGRAPHY OF AUTHORS}

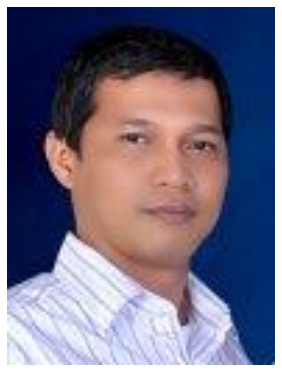

Tengku Yudi Hadiwandra, S.Kom., M.Kom obtained Bachelor Degree in Computer Engineering from Universitas Gunadarma, received Master Degree in Computer Science from Universitas Gadjah Mada. He has been a Lecturer with the Department of Informatics Engineering, Universitas Riau, since 2017. His current research interests include cloud computing, natural language processing, and machine learning.

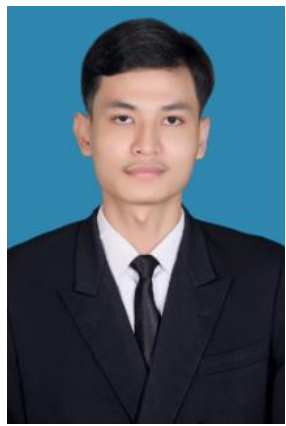

Hariyo Sasongko is currently a student of Bachelor's Degree in Informatics Engineering from Universitas Riau. His current research interests include cloud computing and computer networking. 